Relations industrielles

Industrial Relations

\title{
Job Attitudes: Review of Research and Opinion by F. Herzberg \& al, Pittsburgh, Psychological Service of Pittsburgh, 1957.
}

\section{R. Giroux}

Volume 15, numéro 1, janvier 1960

URI : https://id.erudit.org/iderudit/1022085ar

DOI : https://doi.org/10.7202/1022085ar

Aller au sommaire du numéro

Éditeur(s)

Département des relations industrielles de l’Université Laval

ISSN

0034-379X (imprimé)

1703-8138 (numérique)

Découvrir la revue

Citer ce compte rendu

Giroux, C. (1960). Compte rendu de [Job Attitudes: Review of Research and Opinion by F. Herzberg \& al, Pittsburgh, Psychological Service of Pittsburgh, 1957.] Relations industrielles / Industrial Relations, 15(1), 147-148.

https://doi.org/10.7202/1022085ar

Tous droits réservés @ C Département des relations industrielles de l’Université Laval, 1960
Ce document est protégé par la loi sur le droit d'auteur. L’utilisation des services d'Érudit (y compris la reproduction) est assujettie à sa politique d'utilisation que vous pouvez consulter en ligne.

https://apropos.erudit.org/fr/usagers/politique-dutilisation/ 
téresse le phénomène global de l'industrialisation et la définition du rôle de la direction dans le développement at le déroulement de ce phénomène.

\section{Roger Chartier}

\section{La liberté syndicale, cours d'éducation} ouvriere, Genève, Bureau international du Travail, 1959, 166 pp. (202, rue Queen, Ottawa 4, Ont.) prix: 75 cts.

Le Bureau international du Travail a entrepris la publication d'une série de cours qui s'inscrivent dans le cadre du programme d'éducation ouvrière de l'Organisation internationale du Travail.

Cet ouvrage est consacré à l'importante question de la liberté syndicale. Il est composé de dix-huit leçons groupées en quatre parties. $\mathrm{La}$ première partie retrace à grands traits les étapes principales de l'évolution du droit syndical. La deuxième décrit le rôle joué par l'Organisation internationale du Travail dans le domaine de la liberté syndicale et de la protection du droit syndical. Dans les troisième et quatrième parties, on a examiné un peu plus longuement l'état présent de la liberté syndicale dans les diverses parties du monde. Enfin, dans la dernière leçon, qui constitue la conclusion du cours, les auteurs ont essayé de dégager quelques enseignements de lexamen du problème.

Cet ouvrage se contente de présenter un historique et d'exprimer la situation actuelle en ce qui concerne les libertés syndicales et la protection du droit syndical. Il n'est pas une étude complète de ces questions, mais, en plus d'être une excellente initiation, le plan qu'il utilise peut être très précieux pour ceux qui ont des cours à donner sur le sujet.

Nous recommandons fortement cet ouvrage à tous ceux qui ont à s'occuper d'éducation syndicale.

\section{G. D.}

Job Attitudes: Review of Research and Opinion by $\mathrm{F}$. Herzberg \& al, Pittsburgh, Psychological Service of Pittsburgh, 1957.
Ce volume a pour fin d'étudier, d'organiser et de résumer les travaux publiés depuis 1954 sur le moral et les attitudes des employés envers leur travail. Chaque chapitre se termine par un résumé suivi de questions très pertinentes et de suggestions pour fins de recherche.

Tout d'abord, les auteurs font une revision des analyses sur les caractéristiques des employés mécontents de leur travail: âge, années de service, sexe, éducation, intelligence, personnalité, etc.

Le moral des employés est relié à plusieurs facteurs ou aspects du travail. Pendant les enquêtes, ses divers facteurs sont évalués sous forme de questions différentes quant à la forme, mais semblables quant au contenu. Ici les auteurs ont groupé tous les aspects du travail reliés au moral en dix facteurs élémentaires. Ces facteurs ont une importance relative qui varie selon les circonstances et les conditions de travail. Les auteurs concluent leur étude sur les facteurs en exposant de quelle façon ils sont reliés aux caractéristiques des travailleurs. Leurs suggestions poir fins de recherche sont très originales. Selon eux, il serait bon à ce stage d'utiliser les résultats obtenus directement dans l'industrie, afin d'en vérifier les effets sur la production, le roulement de la main-d'oeuvre, et le moral en général.

Les auteurs abordent également un sujet des plus contreversés: les conséquences directes du moral sur la production. l'absentéisme, le roulement de la main-d'oeuvre, les accidents industriels, la maladie, etc. Les auteurs citent au moins quatorze recherches qui semblent indiquer que le moral des employés est relié positivement à la production. Toutefois, si l'on examine bien les indices de corrélation rapportés dans ces recherches, il est évident que ces corrélations, quoique statistiquement significatives, sont très faibles. La plupart des chercheurs ne rapportent en général aucun indice de validité et de fiabilité sur l'instrument qui a servi à mesurer le moral des employés. Il est bien difficile d'estimer jusqu'à quel point les corrélations sont contaminées, car dans les circonstances il se peut fort bien qu'elles soient dues au hasard. Plus les corrélations sont fai- 
bles, plus il devient impérieux d'exécuter une validation croisée et encore mieux de reprendre les recherches une ou deux fois plus tard: « replication ».

Dans les derniers chapitres, les auteurs analysent les recherches sur les aspects sociaux de la position, les contremaîtres et enfin sur l'hygiène mentale. Le chapitre sur les contremaîtres est des plus révélateurs, surtout l'exposé sur la dualité du rôle de contremaître.

Apres la lecture d'un tel travail, le lecteur est porté à se demander si les chercheurs n'ont pas fait fausse route en étudiant la conjoncture industrielle ¿ partir du moral et des attitudes des employés? Quels progrès ont été enregistrés dans cette voie depuis les dix dernieres années? Pourtant, ces recherches ont été entreprises pour trouver des moyens d'augmenter l'efficacité globale de l'organisation. Quelles découvertes ont abouti à des applications pratiques?

Quelques chercheurs ont compris l'inefficacité de cette méthode d'approche; aussi dans les dernières années les structures mêmes des organisations ont été l'objet de nombreuses analyses. Les résultats obtenus à date laissent entrevoir des recherches encore plus poussées dans cet ordre d'idées. Il convient ici de citer ces quelques phrases de Worthy:

c High employee morale is a by-product of sound organization; it is not a result that can be achieved by and for itself. Good morale among employees and good results from the organization are two aspects of the thing: sound organization and capable leadership $\gg$.

Le présent ouvrage deviendra rapidement un livre de base pour ceux qui font de la recherche dans le domaine des relations humaines.

\section{R. Grroux}

Psychology in Business, by L. Beach, E. L. Clark, New York, McGraw-Hill, 1959.

Les ouvrages de vulgarisation sont assez nombreux en psychologie. D'aucuns sont bien édités et agencés, d'autres le sont moins. Le présent ouvrage est acceptable, à condition de bien dé- terminer à quels lecteurs il s'adresse. Il ne s'adresse pas à l'étudiant qui travaille à l'obtention d'un diplôme en psychologie, mais aux contremaîtres et hommes d'affaires qui se préoccupent de psychologie appliquée et aux étudiants qui sont à la recherche d'une profession.

Ce texte s'est tracé trais objectifs: a) aider le lecteur à comprendre certains principes psychologiques fondamentaux; b) l'amener à appliquer ces principes au monde du travail; c) l'assister dans l'établissement de relations plus gratifiantes dans ses contacts journaliers.

Le contenu du volume est varié. Nous y notons à peu près les mêmes sujets que dans un texte d'introduction à la psychologie. Cependant ces sujets $y$ sont traités de façon bien différente. La définition de la psychologie en tant que science est suivie d'un essai de définition de la normalité. An début du texte, les auteurs partent du développement embryonnaire, et, en passant par le processus de la maturation de l'individu, arrivent à certains sujets propres à la psychologie sociale, comme les responsabilités de l'individu dans la société.

Les auteurs réussissent assez bien à atteindre le premier but de leur travail. Toutefois, leurs recommandations pour aider le lecteur dans son comportement journalier relèvent plus de croyances populaires. Par exemple, ils recommandent de cultiver un sens global de l'humour comme moyen de contrôler l'émotivité.

L'un des auteuns, E.L. Clark, eut certainement à répondre aux nombreuses questions et objections des contremaîtres qui ont assisté aux cours de l'Institut de General Motors. Un sujet très délicat à discuter avec ces genslà est celui de l'évaluation du mérite et de la performance de chaque ermployé. La validité de cette technique de gérance du personnel est constamment battue en brèche par les unions ouvrières. Cette opposition soutenue force les chercheurs à essayer d'établir un critère pour prouver la validité de l'évaluation. Beach et Clark font grand éclat de la nécessité de ce critère. Mais, quelle sorte de critère veulent-ils?' $\mathrm{Si}$ justement nous pou- 